\title{
Essentially high-order compact schemes with application to stochastic volatility models on non-uniform grids
}

\author{
Bertram Düring* Christof Heuer ${ }^{\dagger}$
}

August 3, 2018

\begin{abstract}
We present high-order compact schemes for a linear second-order parabolic partial differential equation (PDE) with mixed second-order derivative terms in two spatial dimensions. The schemes are applied to option pricing PDE for a family of stochastic volatility models. We use a nonuniform grid with more grid-points around the strike price. The schemes are fourth-order accurate in space and second-order accurate in time for vanishing correlation. In our numerical convergence study we achieve fourth-order accuracy also for non-zero correlation. A combination of Crank-Nicolson and BDF-4 discretisation is applied in time. Numerical examples confirm that a standard, second-order finite difference scheme is significantly outperformed.
\end{abstract}

\section{Introduction}

We consider the following parabolic partial differential equation for $u=u\left(x_{1}, x_{2}, t\right)$ in two spatial dimensions and time,

$$
\left.\left.d u_{\tau}+a_{1} u_{x_{1} x_{1}}+a_{2} u_{x_{2} x_{2}}+b_{12} u_{x_{1} x_{2}}+c_{1} u_{x_{1}}+c_{2} u_{x_{2}}=0 \quad \text { in } \Omega \times\right] 0, T\right]=: Q_{T},
$$

subject to suitable boundary conditions and initial condition $u\left(x_{1}, x_{2}, 0\right)=$ $u_{0}\left(x_{1}, x_{2}\right)$ with $T>0$ and $\Omega=\left[x_{\min }^{(1)}, x_{\max }^{(1)}\right] \times\left[x_{\min }^{(2)}, x_{\max }^{(2)}\right] \subset \mathbb{R}^{2}$ with $x_{\min }^{(i)}<$ $x_{\max }^{(i)}$ for $i=1,2$. The functions $a_{i}=a_{i}\left(x_{1}, x_{2}, \tau\right)<0, b_{12}=b_{12}\left(x_{1}, x_{2}, \tau\right)$, $c_{i}=c\left(x_{1}, x_{2}, \tau\right), d=d\left(x_{1}, x_{2}, \tau\right)$ map $Q_{T}$ to $\mathbb{R}$, and $a_{i}(\cdot, \tau), b(\cdot, \tau), c_{i}(\cdot, \tau)$, and $d(\cdot, \tau)$ are assumed to be in $C^{2}(\Omega)$ and $u(\cdot, t) \in C^{6}(\Omega)$ for all $\left.\left.\tau \in\right] 0, T\right]$. We define a uniform spatial grid $G$ with step size $\Delta x_{k}$ in $x_{k}$ direction for $k=1,2$. Setting $f=-d u_{\tau}$ and applying a standard, second-order central difference ap-

\footnotetext{
*Department of Mathematics, University of Sussex, Pevensey II, Brighton, BN1 9QH, United Kingdom, bd80@sussex.ac.uk

${ }^{\dagger}$ Chair of Applied Mathematics / Numerical Analysis, Bergische Universität Wuppertal, Gaußstraße 20, 42097 Wuppertal, Germany, cheuer@uni-wuppertal.de
} 
proximation leads to the elliptic problem

$$
\begin{aligned}
f= & A_{0}-\frac{a_{1}\left(\Delta x_{1}\right)^{2}}{12} \frac{\partial^{4} u}{\partial x_{1}^{4}}-\frac{a_{2}\left(\Delta x_{2}\right)^{2}}{12} \frac{\partial^{4} u}{\partial x_{2}^{4}}-\frac{b_{12}\left(\Delta x_{1}\right)^{2}}{6} \frac{\partial^{4} u}{\partial x_{1}^{3} \partial x_{2}} \\
& -\frac{b_{12}\left(\Delta x_{2}\right)^{2}}{6} \frac{\partial^{4} u}{\partial x_{1} \partial x_{2}^{3}}-\frac{c_{1}\left(\Delta x_{1}\right)^{2}}{6} \frac{\partial^{3} u}{\partial x_{1}^{3}}-\frac{c_{2}\left(\Delta x_{2}\right)^{2}}{6} \frac{\partial^{3} u}{\partial x_{2}^{3}}+\varepsilon
\end{aligned}
$$

with $A_{0}:=a_{1} D_{1}^{c} D_{1}^{c} U_{i_{1}, i_{2}}+a_{2} D_{2}^{c} D_{2}^{c} U_{i_{1}, i_{2}}+b_{12} D_{1}^{c} D_{2}^{c} U_{i_{1}, i_{2}}+c_{1} D_{1}^{c} U_{i_{1}, i_{2}}+c_{2} D_{2}^{c} U_{i_{1}, i_{2}}$, where $D_{k}^{c}$ denotes the central difference operator in $x_{k}$ direction, and $\varepsilon \in \mathcal{O}\left(h^{4}\right)$ if $\Delta x_{k} \in \mathcal{O}(h)$ for $h>0$. We call a finite difference scheme high-order compact (HOC) if its consistency error is of order $\mathcal{O}\left(h^{4}\right)$ for $\Delta x_{1}, \Delta x_{2} \in \mathcal{O}(h)$ for $h>0$, and it uses only points on the compact stencil, $U_{k, p}$ with $k \in\left\{i_{1}-1, i_{1}, i_{1}+1\right\}$ and $p \in\left\{i_{2}-1, i_{2}, i_{2}+1\right\}$, to approximate the solution at $\left(x_{i_{1}}, x_{i_{2}}\right) \in \stackrel{\circ}{G}$.

\section{Auxiliary relations for higher derivatives}

Our aim is to replace the third- and fourth-order derivatives in (2) which are multiplied by second-order terms by equivalent expressions which can be approximated with second order on the compact stencil. Indeed, if we differentiate (1) (using $\left.f=-d u_{\tau}\right)$ once with respect to $x_{k}(k=1,2)$, we obtain relations

$$
\frac{\partial^{3} u}{\partial x_{1}^{3}}=A_{1}, \quad \frac{\partial^{3} u}{\partial x_{2}^{3}}=A_{2},
$$

where we can discretise $A_{i}$ with second order on the compact stencil using the central difference operator. Analogously, we obtain

$$
\begin{array}{rlrl}
\frac{\partial^{4} u}{\partial x_{1}^{4}}=B_{1}-\frac{b_{12}}{a_{1}} \frac{\partial^{4} u}{\partial x_{1}^{3} \partial x_{2}} & \Longleftrightarrow & \frac{\partial^{4} u}{\partial x_{1}^{3} \partial x_{2}}=\frac{a_{1}}{b_{12}} B_{1}-\frac{a_{1}}{b_{12}} \frac{\partial^{4} u}{\partial x_{1}^{4}}, \\
\frac{\partial^{4} u}{\partial x_{2}^{4}}=B_{2}-\frac{b_{12}}{a_{2}} \frac{\partial^{4} u}{\partial x_{1} \partial x_{2}^{3}} \quad \Longleftrightarrow \quad \frac{\partial^{4} u}{\partial x_{1} \partial x_{2}^{3}}=\frac{a_{2}}{b_{12}} B_{2}-\frac{a_{2}}{b_{12}} \frac{\partial^{4} u}{\partial x_{2}^{4}}, \\
\frac{\partial^{4} u}{\partial x_{1}^{3} \partial x_{2}}=C_{1}-\frac{a_{2}}{a_{1}} \frac{\partial^{4} u}{\partial x_{1} \partial x_{2}^{3}} & \Longleftrightarrow & \frac{\partial^{4} u}{\partial x_{1} \partial x_{2}^{3}}=C_{2}-\frac{a_{1}}{a_{2}} \frac{\partial^{4} u}{\partial x_{1}^{3} \partial x_{2}},
\end{array}
$$

where we can approximate $B_{k}$ and $C_{k}$ with second order on the compact stencil using the central difference operator. A detailed derivation can be found in [3, 5].

\section{Derivation of high-order compact schemes}

In general it is not possible to obtain a HOC scheme for (1), since there are four fourth-order derivatives in (2), but only three auxiliary equations for these in (41). Hence, we propose four different versions of the numerical schemes, where only one of the fourth-order derivatives in (2) is left as a second-order remainder term. Using (3) and (4) in (2) we obtain as Version 1 scheme

$$
\begin{aligned}
f= & A_{0}-\frac{c_{1}\left(\Delta x_{1}\right)^{2}}{6} A_{1}-\frac{c_{2}\left(\Delta x_{2}\right)^{2}}{6} A_{2}-\frac{a_{2}\left(\Delta x_{2}\right)^{2}}{12} B_{2}-\frac{b_{12}\left(\Delta x_{2}\right)^{2}}{12} C_{2} \\
& -\frac{a_{1}\left(2 a_{2}\left(\Delta x_{1}\right)^{2}-a_{1}\left(\Delta x_{2}\right)^{2}\right)}{12 a_{2}} B_{1}+\frac{a_{1}\left(a_{2}\left(\Delta x_{1}\right)^{2}-a_{1}\left(\Delta x_{2}\right)^{2}\right)}{12 a_{2}} \frac{\partial^{4} u}{\partial x_{1}^{4}}+\varepsilon,
\end{aligned}
$$


as Version 2 scheme

$$
\begin{aligned}
f= & A_{0}-\frac{c_{1}\left(\Delta x_{1}\right)^{2}}{6} A_{1}-\frac{c_{2}\left(\Delta x_{2}\right)^{2}}{6} A_{2}-\frac{a_{1}\left(\Delta x_{1}\right)^{2}}{12} B_{1}-\frac{b_{12}\left(\Delta x_{1}\right)^{2}}{12} C_{1} \\
& -\frac{a_{2}\left(2 a_{1}\left(\Delta x_{2}\right)^{2}-a_{2}\left(\Delta x_{1}\right)^{2}\right)}{12 a_{1}} B_{2}+\frac{a_{2}\left(a_{1}\left(\Delta x_{2}\right)^{2}-a_{2}\left(\Delta x_{1}\right)^{2}\right)}{12 a_{1}} \frac{\partial^{4} u}{\partial x_{2}^{4}}+\varepsilon
\end{aligned}
$$

as Version 3 scheme

$$
\begin{aligned}
f= & A_{0}-\frac{c_{1}\left(\Delta x_{1}\right)^{2}}{6} A_{1}-\frac{c_{2}\left(\Delta x_{2}\right)^{2}}{6} A_{2}-\frac{a_{1}\left(\Delta x_{1}\right)^{2}}{12} B_{1}-\frac{a_{2}\left(\Delta x_{2}\right)^{2}}{12} B_{2} \\
& -\frac{b_{12}\left(\Delta x_{2}\right)^{2}}{12} C_{2}+\frac{b_{12}\left(a_{1}\left(\Delta x_{2}\right)^{2}-a_{2}\left(\Delta x_{1}\right)^{2}\right)}{12 a_{2}} \frac{\partial^{4} u}{\partial x_{1}^{3} \partial x_{2}}+\varepsilon
\end{aligned}
$$

and, finally, as Version 4 scheme

$$
\begin{aligned}
f= & A_{0}-\frac{c_{1}\left(\Delta x_{1}\right)^{2}}{6} A_{1}-\frac{c_{2}\left(\Delta x_{2}\right)^{2}}{6} A_{2}-\frac{a_{1}\left(\Delta x_{1}\right)^{2}}{12} B_{1}-\frac{a_{2}\left(\Delta x_{2}\right)^{2}}{12} B_{2} \\
& -\frac{b_{12}\left(\Delta x_{1}\right)^{2}}{12} C_{1}+\frac{b_{12}\left(a_{2}\left(\Delta x_{1}\right)^{2}-a_{1}\left(\Delta x_{2}\right)^{2}\right)}{12 a_{1}} \frac{\partial^{4} u}{\partial x_{1} \partial x_{2}^{3}}+\varepsilon
\end{aligned}
$$

Employing the central difference operator with $\Delta x=\Delta y=h$ for $h>0$ to discretise $A_{i}, B_{i}, C_{i}$, in (5)-(8) and neglecting the remaining lower-order term leads to four semi-discrete (in space) schemes. A more detailed description of this approach can be found in [3, 5]. When $a_{1} \equiv a_{2}$ or $b_{12} \equiv 0$ these schemes are fourth-order consistent in space, otherwise second-order.

In time, we apply the implicit BDF4 method on an equidistant time grid with stepsize $k \in \mathcal{O}(h)$. The necessary starting values are obtained using a Crank-Nicolson time discretisation, where we subdivide the first timesteps with a step size $k^{\prime} \in \mathcal{O}\left(h^{2}\right)$ to ensure the fourth-order time discretisation in terms of $h$.

With additional information on the solution of (1) even better results are possible. If the specific combination of pre-factors in (1) and the higher derivatives in the second-order terms is sufficiently small, the second-order term dominates the computational error only for very small step-sizes $h$. Before this error term becomes dominant one can observe a fourth-order numerical convergence. In this case we call the scheme essentially high-order compact (EHOC).

\section{Application to option pricing}

In this section we apply our numerical schemes to an option pricing PDE in a family of stochastic volatility models, with a generalised square root process for the variance with nonlinear drift term,

$$
d S_{t}=\mu S_{t} \mathrm{~d} t+\sqrt{v_{t}} S_{t} \mathrm{~d} W_{t}^{(1)}, \quad d v_{t}=\kappa v_{t}^{\alpha}\left(\theta-v_{t}\right) \mathrm{d} t+\sigma \sqrt{v_{t}} \mathrm{~d} W_{t}^{(2)}
$$

with $\alpha \geq 0$, a correlated, two-dimensional Brownian motion, $d W_{t}^{(1)} d W_{t}^{(2)}=\rho d t$, as well as drift $\mu \in \mathbb{R}$ of the stock price $S$, long run mean $\theta>0$, mean reversion speed $\kappa>0$, and volatility of volatility $\sigma>0$. For $\alpha=0$ one obtains the 
standard Heston model, for $\alpha=1$ the SQRN model, see 11. Using Itô's lemma and standard arbitrage arguments, the option price $V=V(S, v, t)$ solves

$$
\frac{\partial V}{\partial t}+\frac{v S^{2}}{2} \frac{\partial^{2} V}{\partial S^{2}}+\rho \sigma v S \frac{\partial^{2} V}{\partial S \partial v}+\frac{\sigma^{2} v}{2} \frac{\partial^{2} V}{\partial v^{2}}+r S \frac{\partial V}{\partial S}+\kappa v^{\alpha}(\theta-v) \frac{\partial V}{\partial v}-r V=0
$$

where $S, \sigma>0$ and $t \in[0, T[$ with $T>0$. For a European Put with exercise price $K$ we have the final condition $V(S, T)=\max (K-S, 0)$. The transformations $\tau=T-t, u=e^{r \tau} V / K, \hat{S}=\ln (S / K), y=v / \sigma$ as well as $\hat{S}=\varphi(x)$ [2], lead to

$$
\begin{aligned}
\varphi_{x}^{3} u_{\tau}+\frac{\sigma y}{2}\left[\varphi_{x} u_{x x}\right. & \left.+\varphi_{x}^{3} u_{y y}\right]-\rho \sigma y \varphi_{x}^{2} u_{x y} \\
& +\left[\frac{\sigma y \varphi_{x x}}{2}+\left(\frac{\sigma y}{2}-r\right) \varphi_{x}^{2}\right] u_{x}-\kappa \sigma^{\alpha} y^{\alpha} \frac{\theta-\sigma y}{\sigma} \varphi_{x}^{3} u_{y}=0
\end{aligned}
$$

with initial condition $u(x, y, 0)=\max \left(1-e^{\varphi(x)}, 0\right)$. The function $\varphi$ is considered to be four times differentiable and strictly monotone. It is chosen in such a way that grid points are concentrated around the exercise price $K$ in the $S-v$ plane when using a uniform grid in the $x-y$ plane.

Dirichlet boundary conditions are imposed at $x=x_{\min }$ and $x=x_{\max }$ similarly as in [2],

$$
u\left(x_{\min }, y, \tau\right)=u\left(x_{\min }, y, 0\right), \quad u\left(x_{\max }, y, \tau\right)=u\left(x_{\max }, y, 0\right)
$$

for all $\tau \in\left[0, \tau_{\max }\right]$ and $y \in\left[y_{\min }, y_{\max }\right]$. At the boundaries $y=y_{\min }$ and $y=y_{\max }$ we employ the discretisation of the interior spatial domain and extrapolate the resulting ghost-points using

$$
\begin{aligned}
U_{i,-1} & =3 U_{i, 0}-3 U_{i, 1}+U_{i, 2}+\mathcal{O}\left(h^{3}\right), \\
U_{i, M+1} & =3 U_{i, M}-3 U_{i, M-1}+U_{i, M-2}+\mathcal{O}\left(h^{3}\right),
\end{aligned}
$$

for $i=0, \ldots, N$. Third-order extrapolation is sufficient here to ensure overall fourth-order convergence 4 .

\section{$5 \quad$ Numerical experiments}

We employ the function $\varphi(x)=\sinh \left(c_{2} x+c_{1}(1-x)\right) / \zeta$, where $c_{1}=\operatorname{asinh}\left(\zeta \hat{S}_{\min }\right)$, $c_{2}=\operatorname{asinh}\left(\zeta \hat{S}_{\max }\right)$ and $\zeta>0$. We use $\kappa=1.1, \theta=0.2, v=0.3, r=0.05$, $K=100, T=0.25, v_{\min }=0.1, v_{\max }=0.3, S_{\min }=1.5, S_{\max }=250, \rho=0,-0.4$ and $\zeta=7.5$. Hence, $x_{\max }-x_{\min }=y_{\max }-y_{\min }=1$. For the Crank-Nicolson method we use $k^{\prime} / h^{2}=0.4$, for the BDF4 method $k / h=0.1$. We smooth the initial condition according to [6, 3, so that the smoothed initial condition tends towards the original initial condition for $h \rightarrow 0$. We neglect the case $\alpha=0$ (Heston model), since a numerical study of that case has been performed in 2 . In the numerical convergence plots we use a reference solution $U_{\text {ref }}$ on a fine grid $(h=1 / 320)$ and report the absolute $l^{2}$-error compared to $U_{\text {ref. }}$ The numerical convergence order is computed from the slope of the linear least square fit of the points in the log-log plot.

Figure $1(\mathrm{a})$ shows the transformation from $x$ to $S$. The transformation focuses on the region around the strike price. Figures $1(\mathrm{~b}), 1(\mathrm{c}), 1(\mathrm{~d})$ and $1(\mathrm{e})$ show that the HOC schemes lead to a numerical convergence order of about 3.5, 


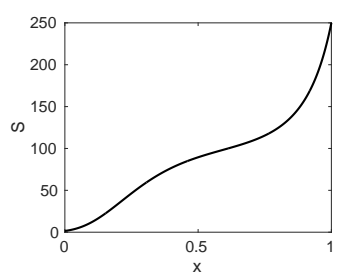

(a) Transformation with $\zeta=7.5$

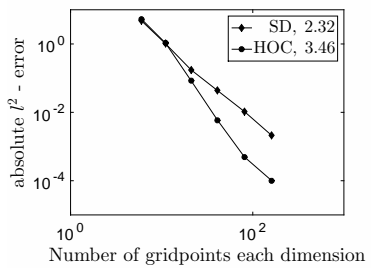

(d) HOC: $\alpha=0.75, \rho=0$

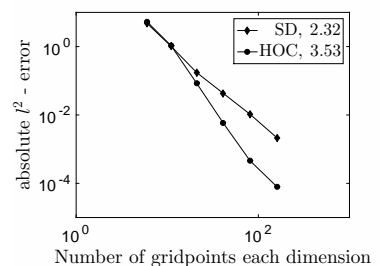

(b) HOC: $\alpha=0.25, \rho=0$

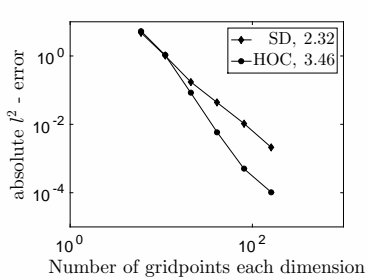

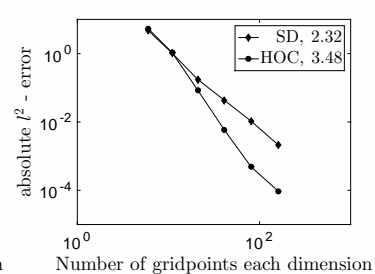

(c) HOC: $\alpha=0.5, \rho=0$

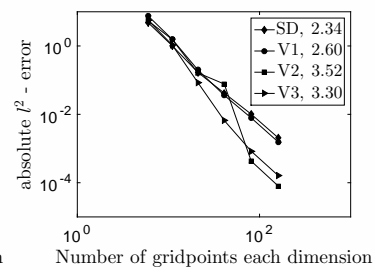

(e) HOC: SQRN model, $\rho=(f)$ EHOC: $\alpha=0.25, \rho=$

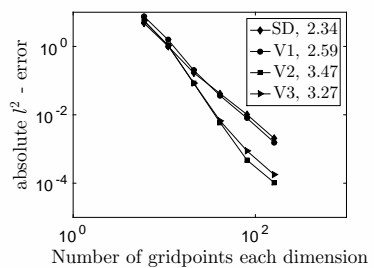
$-0.4$

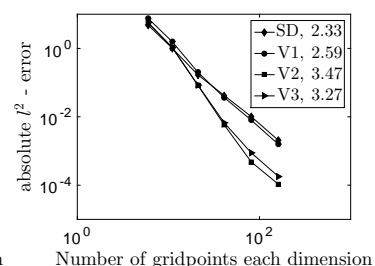

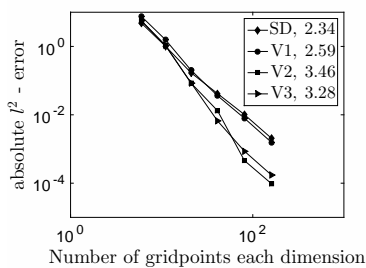

(g) EHOC: $\alpha=0.5, \rho=(\mathrm{h})$ $-0.4$ $-0.4$

Figure 1: Transformation of the spatial grid and numerical convergence plots.

whereas the standard, second-order central difference discretisation (SD) leads to convergence orders of about 2.3 , in the case of vanishing correlation. In all cases with non-vanishing correlation $(\rho \neq 0)$ we observe only slightly improved convergence for Version 1 (V1) when comparing it to the standard discretisation. Version 2 (V2) and Version 3 (V3), however, lead to similar convergence orders as the HOC scheme, even for non-vanishing correlation. Results of Version 4 are not shown as this scheme shows instable behaviour in this example.

In summary, we obtain high-order compact schemes for vanishing correlation and achieve high-order convergence also for non-vanishing correlation for the family (9) of stochastic volatility model. A standard, second-order discretisation is significantly outperformed in all cases.

\section{Acknowledgment}

BD acknowledges support by the Leverhulme Trust research project grant 'Novel discretisations for higher-order nonlinear PDE' (RPG-2015-69). CH was supported by the European Union in the FP7-PEOPLE-2012-ITN Program under Grant Agreement Number 304617 (FP7 Marie Curie Action, Project Multi-ITN STRIKE - Novel Methods in Computational Finance). 


\section{References}

[1] P. Christoffersen, K. Jacobs and K. Mimouni. Models for S\&P500 dynamics: evidence from realized volatility, daily returns, and option prices. Rev. Financ. Stud., 23(8):3141-3189, 2010.

[2] B. Düring, M. Fournié and C. Heuer. High-order compact finite difference schemes for option pricing in stochastic volatility models on non-uniform grids. J. Comput. Appl. Math., 271(18):247-266, 2014.

[3] B. Düring and C. Heuer. High-order compact schemes for parabolic problems with mixed derivatives in multiple space dimensions. SIAM J. Numer. Anal., 53(5):2113-2134, 2015.

[4] B. Gustafsson. The convergence rate for difference approximations to general mixed initial-boundary value problems. SIAM J. Numer. Anal., 18(2):179190, 1981.

[5] C. Heuer. High-order compact finite difference schemes for parabolic partial differential equations with mixed derivative terms and applications in computational finance. PhD thesis, University of Sussex, August 2014. http://sro.sussex.ac.uk/49800/

[6] H.O. Kreiss, V. Thomee and O. Widlund. Smoothing of initial data and rates of convergence for parabolic difference equations. Commun. Pure Appl. Math., 23:241-259, 1970. 